\title{
Rieckmann, Marco
}

\section{Die Bedeutung von Bildung für nachhaltige Entwicklung für das Erreichen der Sustainable Development Goals (SDGs) \\ ZEP : Zeitschrift für internationale Bildungsforschung und Entwicklungspädagogik 41 (2018) 2, S. 4-10}

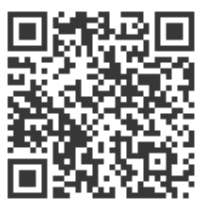

Quellenangabe/ Reference:

Rieckmann, Marco: Die Bedeutung von Bildung für nachhaltige Entwicklung für das Erreichen der Sustainable Development Goals (SDGs) - In: ZEP : Zeitschrift für internationale Bildungsforschung und Entwicklungspädagogik 41 (2018) 2, S. 4-10 - URN: urn:nbn:de:0111-pedocs-189555 - DOI: 10.25656/01:18955

https://nbn-resolving.org/urn:nbn:de:0111-pedocs-189555

https://doi.org/10.25656/01:18955

in Kooperation mit / in cooperation with:

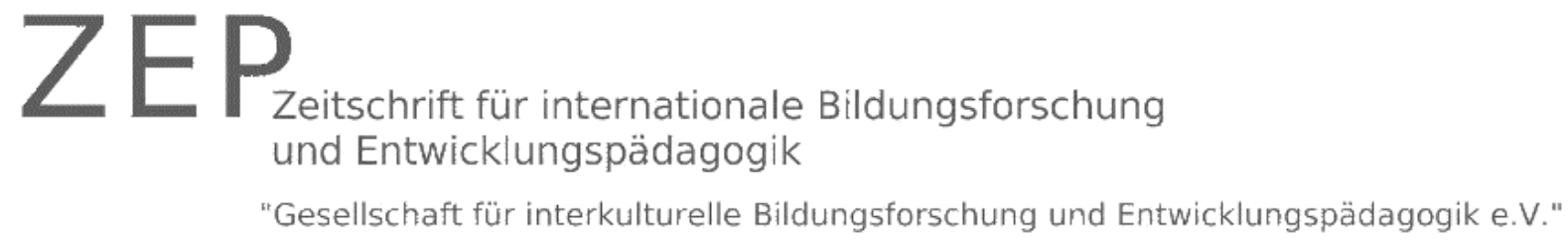

http://www.uni-bamberg.de/allgpaed/zep-zeitschrift-fuer-internationale-bildungsforschung-und-entwicklungspaedagogik/profil

\section{Nutzungsbedingungen}

Gewährt wird ein nicht exklusives, nicht übertragbares, persönliches und beschränktes Recht auf Nutzung dieses Dokuments. Dieses Dokument ist ausschließlich für den persönlichen, nicht-kommerziellen Gebrauch bestimmt. Die Nutzung stellt keine Übertragung des Eigentumsrechts an diesem Dokument dar und gilt vorbehaltlich der folgenden Einschränkungen Auf sämtlichen Kopien dieses Dokuments müssen alle Urheberrechtshinweise und sonstigen Hinweise auf gesetzlichen Schutz beibehalten werden. Sie dürfen dieses Dokument nicht in irgendeiner Weise abändern, noch dürfen Sie dieses Dokument für öffentliche oder kommerzielle Zwecke vervielfältigen, öffentlich ausstellen, aufführen, vertreiben oder anderweitig nutzen.

Mit der Verwendung dieses Dokuments erkennen Sie die Nutzungsbedingungen an.

\section{Terms of use}

We grant a non-exclusive, non-transferable, individual and limited right to using this document

This document is solely intended for your personal, non-commercial use. Use of this document does not include any transfer of property rights and it is conditional to the following limitations: All of the copies of this documents must retain all copyright information and other information regarding legal protection. You are not allowed to alter this document in any way, to copy it for public or commercial purposes, to exhibit the document in public, to perform, distribute or otherwise use the document in public.

By using this particular document, you accept the above-stated conditions of use.

\section{Kontakt / Contact:}

peDOCS

DIPF | Leibniz-Institut für Bildungsforschung und Bildungsinformation Informationszentrum (IZ) Bildung

E-Mail: pedocs@dipf.de

Internet: www.pedocs.de

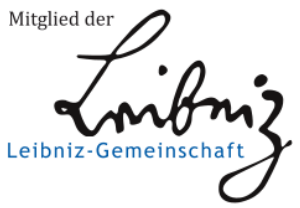



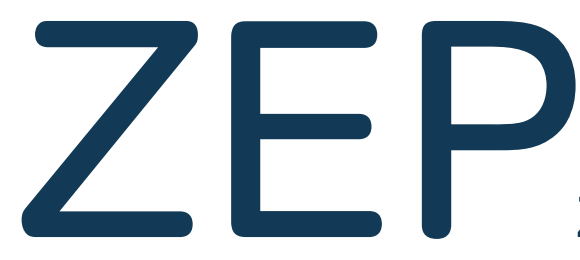

Zeitschrift für internationale Bildungsforschung und Entwicklungspädagogik

Mit: Mitteilungen der DGfE-Kommission

Vergleichende und Internationale

Erziehungswissenschaft

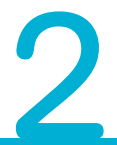

Sustainable • Die Bedeutung von BNE für das Erreichen der Sustainable Development

Development Goals (SDGs)

Goals • Die Bildungsagenda der Vereinten Nationen aus dem Blick der internationalen Bildungsforschung

- Die Umsetzung der SDGs im deutschen Schulsystem

- Teaching the Sustainable Development Goals - Geschichten des Wandels

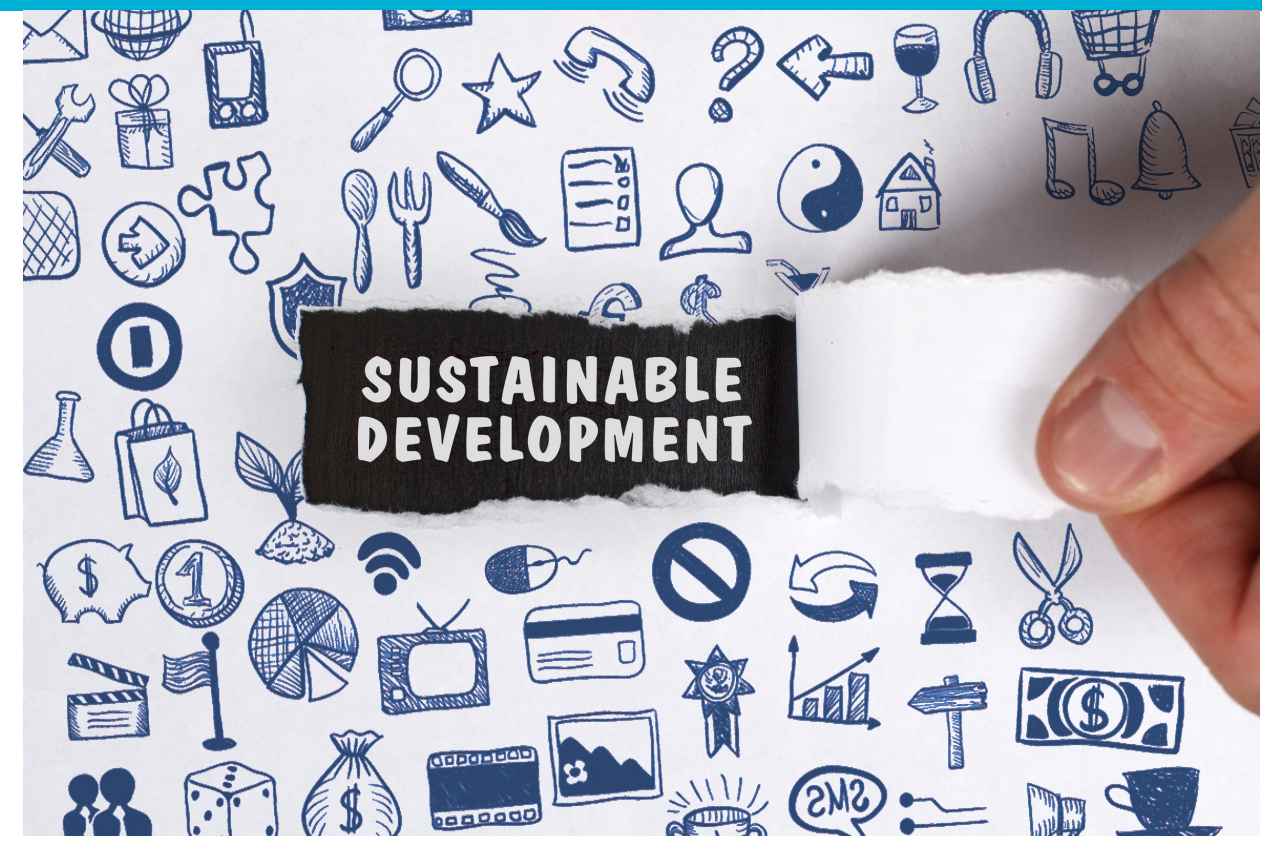


A m 25. September 2015 verabschiedete die UN-Vollversammlung die Agenda 2030 für Nachhaltige Entwicklung mit 17 Nachhaltigkeitszielen (Sustainable Development Goals - SDGs). Diese Ziele beschreiben wichtige Entwicklungsherausforderungen, um für alle jetzt und in Zukunft ein nachhaltiges, friedliches, wohlhabendes und gerechtes Leben zu sichern. Ein wesentliches Merkmal der Agenda 2030 ist ihre Unteilbarkeit und Universalität. Sie richtet sich gleichermaßen an den Globalen Süden und Norden.

Im Ziel 4.7 des SDG 4 zu hochwertiger Bildung werden Bildung für nachhaltige Entwicklung (BNE) und Global Citizenship Education (GCED) ausdrücklich als relevante Bildungskonzepte anerkannt. Gleichzeitig gilt es, die zentrale Bedeutung von BNE für alle anderen 16 SDGs zu betonen: BNE soll allen Personen ermöglichen, zur Erreichung der SDGs beizutragen, indem sie die notwendigen Kenntnisse über (nicht-)nachhaltige Entwicklungen und Kompetenzen erwerben können, die sie nicht nur brauchen, um zu verstehen, worum es bei den SDGs geht, sondern auch, um sich als informierte Bürger/innen aktiv für die Herbeiführung der notwendigen gesellschaftlichen Transformation einzusetzen. Es geht dabei nicht nur allein darum, auf die existenten Problemlagen reagieren zu können, sondern vor allem darum, vorausschauend mit Zukunft umzugehen sowie an innovativen Lebens- und Gesellschaftsentwürfen mitzuwirken, die einen zukunftsweisenden und verantwortlichen Übergang in eine nachhaltige Welt möglich machen.

\section{Impressum}

ZEP - Zeitschrift für internationale Bildungsforschung und Entwicklungspädagogik ISSN 1434-4688

\section{Herausgeber:}

Gesellschaft für interkulturelle Bildungsforschung und Entwicklungspädagogik e.V. und KommEnt

Schriftleitung: Annette Scheunpflug/

Claudia Bergmüller

\section{Technische Redaktion:}

Claudia Bergmüller (verantwortlich) 0951/863-

1832, Markus Ziebarth (Rezensionen),

Anne-Christine Banze (Schlaglichter)

\section{Redaktionsanschrift:}

ZEP-Redaktion, Lehrstuhl Allgemeine

Pädagogik, Markusplatz 3, 96047 Bamberg

\section{Verlag:}

Waxmann Verlag GmbH, Steinfurter Straße 555 48159 Münster, Tel.: 0251/26 5040

E-Mail:info@waxmann.com
Zielsetzung dieses Themenheftes ist es, eine Einführung in die SDGs, insbesondere das Bildungs-SDG, und die Bedeutung von Bildungs- und Lernprozessen für die SDGs zu geben. Zudem wird beleuchtet, inwiefern in Deutschland die SDGs bereits Eingang in das Bildungssystem gefunden haben.

Marco Rieckmann befasst sich mit der Bedeutung von BNE für das Erreichen der SDGs. Dabei wird deutlich, dass BNE die Entwicklung von Nachhaltigkeitskompetenzen fördern kann, die Individuen dazu befähigen, an der Umsetzung der SDGs mitzuwirken. Gleichzeitig kann BNE auch spezifische Lernprozesse ermöglichen, die dazu benötigt werden, sich ein bestimmtes SDG zu erschließen und eigene Beiträge zu diesem Ziel zu leisten.

Christel Adick befasst sich mit der Bildungsagenda der Vereinten Nationen bis zum Jahr 2030 aus dem Blickwinkel der internationalen Bildungsforschung. Zunächst werden die SDGs mit anderen UN-Programmen seit den 1990er Jahren historisch kontextualisiert, um dadurch die Bedeutsamkeit des SDG 4 besser einordnen zu können. Zudem werden die zehn Unterpunkte des SDG 4 hinsichtlich ihrer Aussagen zu den angestrebten Entwicklungen analysiert. Beleuchtet wird außerdem die inzwischen im System der Vereinten Nationen begonnene Arbeit an Indikatoren, mit denen die Realisierung des SDG 4 jährlich weltweit erhoben und verglichen werden soll. Abschließend wird die Anschlussfähigkeit des SDG 4-Monitoring an Forschung und Lehre im Rahmen einer internationalen Bildungsforschung reflektiert.
Georg Müller-Christ, Bror Giesenbauer und Merle Katrin Tegeler stellen Ergebnisse einer Studie im Auftrag des Rats für nachhaltige Entwicklung der Bundesregierung zur Umsetzung der SDGs im deutschen Bildungssystem vor. Auf Basis von Experteninterviews und Online-Recherchen werden sechs Bildungsbereiche einzeln beleuchtet: Frühkindliche Bildung, Schule, berufliche Bildung, Hochschule, non-formales und informelles Lernen sowie Fort- und Weiterbildung. Es zeigt sich, dass die SDGs bislang v.a. dort transportiert werden, wo Umweltbildung, Globales Lernen oder BNE bereits Pfade angelegt haben. Gleichwohl scheint der Ordnungsrahmen der SDGs den bereits Aktiven Schwung zu geben, an der Umsetzung einer nachhaltigen Entwicklung weiterzuarbeiten und ihre Aktivitäten ansprechend darzustellen.

Das Lehrmaterial „Teaching the Sustainable Development Goals" stellt Thomas Hoffmann vor. Es ist 2017 im Rahmen des interkulturell konzipierten ESD Expert Net Mexico, India, South Africa and Germany entstanden. Eine Besonderheit des Materials ist, dass hier positive Erzählungen des Wandels genutzt werden, um zu zeigen, dass die SDGs erreicht werden können.

Zudem erhalten Sie auch in dieser Ausgabe Informationen zum Globalen Lernen und zur internationalen Bildungsforschung etwa in Form von Rezensionen und Veranstaltungshinweisen.

Eine anregende Lektüre wünschen Achim Beule und Marco Rieckmann Stuttgart/Vechta, Juli 2018 doi.org/10.31244/zep.2018.02.01

Begründet von: Alfred K. Treml (†) mit dem AK Dritte Welt Reutlingen.

Ehemals in der Redaktion: Barbara Asbrand, Hans Gängler, Sigrid Görgens, Richard Helbling (Schweiz), Linda Helfrich, Alfred Holzbrecher, Torsten Jäger, Gerhard Mersch, Renate Nestvogel, Gottfried Orth, Georg Friedrich Pfäfflin, Arno Schöppe, Birgit Schößwender, Horst Siebert, Klaus-Jürgen Tillmann, Barbara Toepfer, Erwin Wagner, Joachim Winter.

Aktuell in der Redaktion: Achim Beule, Claudia Bergmüller, Hans Bühler, Christian Brüggemann, Asit Datta, Julia Franz, Norbert Frieters-Reermann, Heidi Grobbauer (Österreich), Helmuth Hartmeyer (Österreich), Susanne Höck, Karola Hoffmann, Lydia Kater-Wettstädt, Ulrich Klemm, Gregor LangWojtasik, Sarah Lange, Volker Lenhart, Claudia Lohrenscheit, Bernd Overwien, Marco Rieckmann, Annette Scheunpflug, Klaus Seitz, Susanne Timm, Rudolf Tippelt

Anzeigenverwaltung: Waxmann Verlag GmbH, Maike Rösmann: roesmann@waxmann.com

Abbildungen: (Falls nicht bezeichnet) Privatfotos oder Illustrationen der Autor/inn/en

Titelbild: (CEgor, www.stock.adobe.com

Erscheinungsweise und Bezugsbedingungen: erscheint vierteljährlich; Jahresabonnement EUR 24,00, Einzelheft EUR 11,00; alle Preise verstehen sich zuzüglich Versandkosten; zu beziehen durch alle Buchhandlungen oder direkt vom Verlag. Abbestellungen spätestens acht Wochen vor Ablauf des Jahres. Das Heft ist auf umweltfreundlichem chlorfreien Papier gedruckt. Diese Publikation ist gefördert von Brot für die Welt - Evangelischen Entwicklungsdienst, Referat für Inlandsförderung, Berlin. 
Zeitschrift für internationale Bildungsforschung und Entwicklungspädagogik

Mit: Mitteilungen der DGfE-Kommission

Vergleichende und Internationale Erziehungswissenschaft

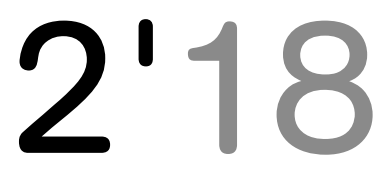

\section{Marco Rieckmann}

Themen 4 Die Bedeutung von Bildung für nachhaltige Entwicklung für das Erreichen der Sustainable Development Goals (SDGs)

Christel Adick

11 Die Bildungsagenda der Vereinten Nationen aus dem

Blickwinkel der internationalen Bildungsforschung

Georg Müller-Christ / Bror Giesenbauer / Merle Katrin Tegeler

19 Die Umsetzung der SDGs im deutschen Bildungssystem Studie im Auftrag des Rats für nachhaltige Entwicklung der Bundesregierung

\section{Thomas Hoffmann}

27 Teaching the Sustainable Development Goals Geschichten des Wandels

Porträt 35 Zwei Angebote für einen besseren Materialüberblick

VENRO 37 BMZ-Wirkungsstudie in der Inlandsarbeit und VENRO-Begleitprojekt

VIE 38 Faire Schule meets SDG-Labor/Hochwertige Bildung für Alle/ GloNet - das Netzwerk für Globales Lernen in der beruflichen Bildung

41 Rezensionen

43 Schlaglichter 


\section{Die Bedeutung von Bildung für nachhaltige Entwicklung für das Erreichen der Sustainable Development Goals (SDGs)}

\begin{abstract}
Zusammenfassung
Die 2015 von den Vereinten Nationen verabschiedeten Sustainable Development Goals (SDGs) zielen darauf, für alle, jetzt und in Zukunft, ein nachhaltiges, friedliches, wohlhabendes und gerechtes Leben zu ermöglichen. Für das Erreichen der SDGs kommt Bildung für nachhaltige Entwicklung (BNE) eine wichtige Bedeutung zu. BNE kann die Entwicklung von Nachhaltigkeitskompetenzen fördern, die Individuen nicht nur dazu befähigen, die SDGs in ihrer ganzen Komplexität zu verstehen, sondern auch eigene Beiträge zum Erreichen der Ziele zu leisten. Gleichzeitig kann BNE auch spezifische Lernprozesse ermöglichen, die dazu benötigt werden, sich ein bestimmtes SDG zu erschließen und eigene Beiträge zu diesem Ziel zu leisten. Dazu bietet der von der UNESCO veröffentliche Leitfaden „Education for Sustainable Development Goals. Learning Objectives“ vielfältige Anregungen. Für die Entwicklung der Nachhaltigkeitskompetenzen und eine Auseinandersetzung mit den einzelnen SDGs bedarf es einer handlungsorientierten, transformativen Pädagogik.
\end{abstract}

Schlüsselworte: Bildung für nachhaltige Entwicklung, Sustainable Development Goals (SDGs), transformative Pädagogik

\footnotetext{
Abstract

The Sustainable Development Goals (SDGs), adopted by the United Nations in 2015, aim to provide a sustainable, peaceful, prosperous and just life for all, now and in the future. Education for Sustainable Development (ESD) plays an important role in achieving the SDGs. ESD can foster the development of sustainability competencies that not only empower individuals to understand the full complexity of the SDGs, but also to make their own contribution to achieving the goals. At the same time, ESD can also facilitate specific learning outcomes needed to understand a specific SDG and to contribute to achieving that goal. The guide "Education for Sustainable Development Goals. Learning Objectives", published by the UNESCO, offers diverse suggestions for such learning outcomes and how to foster them. For the development of the sustainability competencies and
}

learning outcomes related to specific SDGs, an action-oriented, transformative pedagogy is needed.

Keywords: Education for sustainable development, Sustainable Development Goals (SDGs), transformative pedagogy

\section{Die Nachhaltigkeitsziele - eine ambitionierte und universelle Agenda für die Transformation der Weltgesellschaft}

Am 25. September 2015 verabschiedete die UN-Vollversammlung die Agenda 2030 für Nachhaltige Entwicklung (Vereinte Nationen, 2015). Dieses neue globale Rahmenwerk zur nachhaltigen Entwicklung der Weltgesellschaft wurde im Anschluss an die UN-Konferenz über nachhaltige Entwicklung (Rio + 20) in Rio de Janeiro (Brasilien) im Juni 2012 in einem dreijährigen Prozess entwickelt, an dem neben den UN-Mitgliedstaaten, NGOs und anderen Akteuren auch Millionen von Menschen aus der ganzen Welt beteiligt waren (Kercher, 2015; Scholz, 2015, 2017). Im Mittelpunkt der Agenda 2030 stehen 17 Nachhaltigkeitsziele (Sustainable Development Goals, SDGs).

Die universellen, transformativen und inklusiven SDGs beschreiben wichtige Entwicklungsherausforderungen für die Menschheit. Das Ziel der 17 SDGs (Abb. 1) ist es, für alle, jetzt und in Zukunft, ein nachhaltiges, friedliches, wohlhabendes und gerechtes Leben zu ermöglichen. Die SDGs beziehen sich auf globale Herausforderungen, die für das Überleben der Menschheit entscheidend sind. Sie setzen ökologische Grenzen fest und definieren kritische Schwellenwerte für den Einsatz natürlicher Ressourcen. Sie erkennen an, dass Armutsbekämpfung Hand in Hand mit Strategien gehen muss, die wirtschaftliche Entwicklung befördern. Sie widmen sich einer Reihe von sozialen Bedürfnissen, einschließlich Bildung, Gesundheit, sozialer Absicherung und Beschäftigungsmöglichkeiten, während sie gleichzeitig auf die Bewältigung des Klimawandels und Umweltschutz abzielen. Die SDGs adressieren die wichtigsten systemischen Barrieren für eine nachhaltige Entwicklung wie Ungleichheit, nicht-nachhaltige Konsummuster, schwache institutionelle Kapazitäten und Umweltzerstörung (Messner \& Scholz, 2015; Scholz, 2015, 2017). 
1. Keine Armut - Armut in jeder Form und überall beenden

2. Keine Hungersnot - Den Hunger beenden, Ernährungssicherheit und eine bessere Ernährung erreichen und eine nachhaltige Landwirtschaft fördern

3. Gute Gesundheitsversorgung und Wohlergehen - Ein gesundes Leben für alle Menschen jeden Alters gewährleisten und ihr Wohlergehen fördern

4. Hochwertige Bildung - Inklusive, gerechte und hochwertige Bildung gewährleisten und Möglichkeiten des lebenslangen Lernens für alle fördern

5. Gleichberechtigung der Geschlechter - Geschlechtergerechtigkeit und Selbstbestimmung für alle Frauen und Mädchen erreichen

6. Sauberes Wasser und sanitäre Einrichtungen - Verfügbarkeit und nachhaltige Bewirtschaftung von Wasser und Sanitärversorgung für alle gewährleisten

7. Erschwingliche und saubere Energie - Zugang zu bezahlbarer, verlässlicher, nachhaltiger und zeitgemäßer Energie für alle sichern

8. Gute Arbeitsplätze und wirtschaftliches Wachstum - Dauerhaftes, inklusives und nachhaltiges Wirtschaftswachstum, produktive Vollbeschäftigung und menschenwürdige Arbeit für alle fördern

9. Industrie, Innovation und Infrastruktur - Eine belastbare Infrastruktur aufbauen, inklusive und nachhaltige Industrialisierung fördern und Innovationen unterstützen

10. Reduzierte Ungleichheiten - Ungleichheit innerhalb von und zwischen Staaten verringern

11. Nachhaltige Städte und Gemeinden - Städte und Siedlungen inklusiv, sicher, widerstandsfähig und nachhaltig machen

12. Verantwortungsvoller Konsum und Produktion - Für nachhaltige Konsum- und Produktionsmuster sorgen

13. Klimaschutz - Umgehend Maßnahmen zur Bekämpfung des Klimawandels und seiner Auswirkungen ergreifen

14. Leben im Wasser - Ozeane, Meere und Meeresressourcen im Sinne einer nachhaltigen Entwicklung erhalten und nachhaltig nutzen

15. Leben an Land - Landökosysteme schützen, wiederherstellen und ihre nachhaltige Nutzung fördern, Wälder nachhaltig bewirtschaften, Wüstenbildung bekämpfen, Bodenverschlechterung stoppen und umkehren und den Biodiversitätsverlust stoppen

16. Frieden, Gerechtigkeit und starke Institutionen - Friedliche und inklusive Gesellschaften im Sinne einer nachhaltigen Entwicklung fördern, allen Menschen Zugang zur Justiz ermöglichen und effektive, rechenschaftspflichtige und inklusive Institutionen auf allen Ebenen aufbauen

17. Partnerschaften für die Ziele - Umsetzungsmittel stärken und die globale Partnerschaft für nachhaltige Entwicklung wiederbeleben

Quelle: http://www.bmz.delde/ministerium/ziele/2030_agenda/17_ziele/index.html

Damit die Ziele erreicht werden können, müssen viele ihren Beitrag leisten: Regierungen, die Privatwirtschaft, die Zivilgesellschaft und alle Menschen auf der ganzen Welt. Es wird von den Regierungen erwartet, dass sie sich die Ziele zu eigen machen und nationale Rahmenbedingungen sowie politische Strategien und Maßnahmen zur Umsetzung der Agenda 2030 schaffen.

Ein wesentliches Merkmal der Agenda 2030 ist ihre Universalität und Unteilbarkeit. Sie richtet sich an alle Länder - aus dem
Globalen Süden und dem Globalen Norden. Alle Länder, die sich der Agenda 2030 verschreiben, müssen ihre eigenen Entwicklungsbemühungen an dem Ziel ausrichten, den Wohlstand zu fördern und gleichzeitig den Planeten zu schützen, um eine nachhaltige Entwicklung zu erreichen (Messner \& Scholz, 2015). Insofern können in Bezug auf die SDGs alle Länder als Entwicklungsländer betrachtet werden, und alle Länder müssen dringende Maßnahmen zur Förderung einer nachhaltigen Entwicklung ergreifen.

\section{Bildung für nachhaltige Entwicklung - ein Schlüsselinstrument für das Erreichen der Nachhaltigkeitsziele}

\author{
"Education can, and must, contribute to a new vision of \\ sustainable global development." (UNESCO, 2015b, S. 32)
}

Den Weg einer nachhaltigen Entwicklung einzuschlagen, wird eine tiefgreifende Transformation unserer Art zu Denken und zu Handeln erfordern. Um eine nachhaltigere Welt zu schaffen und mit nachhaltigkeitsrelevanten Problemstellungen umzugehen, wie sie in den SDGs beschrieben werden, müssen Individuen Change Agents für eine nachhaltige Entwicklung werden. Sie benötigen das Wissen, die Fähigkeiten, die Werte und die Einstellungen, die sie dazu in die Lage versetzen, zu einer nachhaltigen Entwicklung beizutragen. Bildung ist daher entscheidend für das Erreichen einer nachhaltigen Entwicklung. Doch nicht jede Art von Bildung unterstützt eine nachhaltige Entwicklung. Bildung, die das Wirtschaftswachstum allein fördert, kann auch zu einer Zunahme nicht nachhaltiger Konsummuster führen. Das mittlerweile gut etablierte Konzept einer Bildung für nachhaltige Entwicklung (BNE) ermächtigt die Lernenden, fundierte Entscheidungen für den Erhalt der Umwelt, wirtschaftliche Leistungsfähigkeit und eine gerechte Gesellschaft für gegenwärtige und zukünftige Generationen zu treffen und entsprechend verantwortungsbewusst zu handeln (Rieckmann, 2016). BNE zielt darauf ab, Kompetenzen zu entwickeln, die es den Individuen ermöglichen, über ihre eigenen Handlungen unter Berücksichtigung ihrer gegenwärtigen und zukünftigen sozialen, kulturellen, wirtschaftlichen und ökologischen Auswirkungen - unter lokaler und globaler Perspektive - nachzudenken, in komplexen Situationen nachhaltig zu handeln, was von dem Individuum erfordern kann, neue Richtungen einzuschlagen und sich an gesellschaftspolitischen Prozessen zu beteiligen, die ihre Gesellschaften in Richtung einer nachhaltigen Entwicklung bewegen (de Haan, 2010; de Haan et al., 2008; Overwien, 2013; Rieckmann, 2013, 2016, 2018).

BNE muss als integraler Bestandteil hochwertiger Bildung verstanden werden, die ein Element des Konzepts lebenslangen Lernens ist: Alle Bildungseinrichtungen - vom Vorschul- bis zum Hochschulbereich bis hin zur non-formalen und informellen Bildung - können und sollten es als ihre Verantwortung betrachten, sich intensiv mit Fragen einer nachhaltigen Entwicklung zu beschäftigen und die Entwicklung von Nachhaltigkeitskompetenzen zu fördern (Nationale Plattform Bildung für nachhaltige Entwicklung, 2017; Rieckmann, 2016). BNE ermöglicht Bildungs- und Lernprozesse, die für jeden Lernenden angesichts der heutigen Herausforderungen sehr relevant sind. 
Die internationale Anerkennung von BNE als wichtiges Instrument für die Förderung einer nachhaltigen Entwicklung ist stetig gewachsen. Diese Bedeutung von BNE wurde auch auf den drei wichtigsten globalen Gipfeltreffen für nachhaltige Entwicklung betont: die UN-Konferenz über Umwelt und Entwicklung (UNCED) 1992 in Rio de Janeiro, der Weltgipfel für Nachhaltige Entwicklung (WSSD) 2002 in Johannesburg und die UN-Konferenz für Nachhaltige Entwicklung (UNCSD) 2012 ebenfalls in Rio de Janeiro. Die UN-Dekade Bildung für nachhaltige Entwicklung (2005-2014) zielte darauf ab, die Prinzipien und Praktiken einer nachhaltigen Entwicklung umfassend in Bildungs- und Lernprozesse zu integrieren, um Veränderungen von Wissen, Werten und Einstellungen im Sinne der Vision einer nachhaltigeren und gerechteren Gesellschaft für alle zu fördern. Das Weltaktionsprogramm (WAP) Bildung für nachhaltige Entwicklung, das am 12. November 2014 auf der UNESCO-Weltkonferenz zu BNE (Aichi-Nagoya, Japan) lanciert wurde, strebt danach, auf der Grundlage der Ergebnisse der Dekade, die Umsetzung von BNE zu intensivieren (UNESCO, 2014).

BNE wird in den SDGs ausdrücklich als Teil des Ziels 4.7 des SDG zu hochwertiger Bildung anerkannt (Abb. 2) - zusammen mit Global Citizenship Education (GCED), die die UNESCO als einen komplementären Ansatz fördert (UNESCO, 2015a).

\section{Abb. 2: Ziel 4.7 der SDGs}

Bis 2030 sicherstellen, dass alle Lernenden die notwendigen Kenntnisse und Qualifikationen zur Förderung nachhaltiger Entwicklung erwerben, unter anderem durch Bildung für nachhaltige Entwicklung und nachhaltige Lebensweisen, Menschenrechte, Geschlechtergleichstellung, eine Kultur des Friedens und der Gewaltlosigkeit, Weltbürgerschaft und die Wertschätzung kultureller Vielfalt und des Beitrags der Kultur zu nachhaltiger Entwicklung.

Quelle: Vereinte Nationen, 2015, S. 19

Gleichzeitig ist es wichtig, die zentrale Bedeutung von BNE für alle anderen 16 SDGs zu betonen. Mit ihrem Ziel, übergreifende Nachhaltigkeitskompetenzen bei den Lernenden zu entwickeln, leistet BNE einen wesentlichen Beitrag für alle Bemühungen zum Erreichen der SDGs. Denn BNE befähigt Individuen, an einer nachhaltigen Entwicklung mitzuwirken, indem sie den gesellschaftlichen, wirtschaftlichen und politischen Wandel fördern und ihr eigenes Verhalten ändern. BNE kann spezifische kognitive, sozioemotionale und verhaltensbezogene Lernergebnisse erzielen, die es Individuen ermöglichen, mit den besonderen Herausforderungen jedes SDG umzugehen und so dessen Umsetzung zu unterstützen. BNE ermöglicht es allen Individuen, zur Erreichung der SDGs beizutragen, indem sie mit den notwendigen Kenntnissen und Kompetenzen ausgestattet werden, die sie nicht nur brauchen, um zu verstehen, worum es bei den SDGs geht, sondern auch um sich als informierte Bürgerinnen und Bürger für die Herbeiführung der notwendigen Transformation einzusetzen (UNESCO, 2017).

\section{Lernziele für das Erreichen des SDGs}

BNE kann übergreifende Nachhaltigkeitskompetenzen entwickeln, die für alle SDGs relevant sind. Zudem kann BNE auch spezifische Lernergebnisse erzielen, die wir benötigen, um zum Erreichen eines bestimmten SDGs beizutragen.

Schlüsselkompetenzen für das Erreichen der SDGs Die Weltgesellschaft steht vor vielen Herausforderungen: eine zunehmende Komplexität und Unsicherheit, mehr Individualisierung und soziale Vielfalt, wachsende wirtschaftliche und kulturelle Einheitlichkeit, eine Verschlechterung der Ökosystemdienstleistungen sowie eine zunehmende Anfälligkeit und Exposition gegenüber natürlichen und technologischen Gefahren. Vor diesem Hintergrund müssen die Menschen lernen, die komplexe Welt zu verstehen, in der sie leben. Sie müssen in der Lage sein, zusammenzuarbeiten, ihre Meinung zu äußern und sich für positive Veränderungen einzusetzen (UNESCO, 2015b). Es geht darum, „Nachhaltigkeitsbürger*innen“ (Rieckmann \& Schank, 2016; Wals, 2015; Wals \& Lenglet, 2016) zu werden. „Nachhaltigkeitsbürger*innen“ benötigen bestimmte Schlüsselkompe-

\section{Abb. 3: Nachhaltigkeitskompetenzen}

- Kompetenz zum vernetzten Denken: die Fähigkeiten, Zusammenhänge zu erkennen und zu verstehen; komplexe Systeme zu analysieren; zu überlegen, wie Systeme in verschiedene Domänen und verschiedene Skalen eingebettet sind; und mit Unsicherheit umzugehen Kompetenz zum vorausschauenden Denken: die Fähigkeiten, multiple (mögliche, wahrscheinliche und wünschenswerte) Zukünfte zu verstehen und zu bewerten; eigene Visionen für die Zukunft zu schaffen; das Vorsorgeprinzip anzuwenden; die Konsequenzen von Handlungen zu beurteilen; und mit Risiken und Veränderungen umzugehen Normative Kompetenz: die Fähigkeiten, die Normen und Werte zu verstehen und zu reflektieren, die den eigenen Handlungen zugrunde liegen; und Nachhaltigkeitswerte, Prinzipien und Ziele im Kontext von Interessenkonflikten und Trade-offs, unsicheren Kenntnissen und Widersprüchen zu verhandeln

Strategische Kompetenz: die Fähigkeiten zur kollektiven Entwicklung und Umsetzung innovativer Maßnahmen, die Nachhaltigkeit auf lokaler Ebene und darüber hinaus voranbringen

- Kooperationskompetenz: die Fähigkeiten, von anderen zu lernen; die Bedürfnisse, Perspektiven und Handlungen anderer zu verstehen und zu respektieren (Empathie), eine Beziehung zu ihnen aufzubauen und für sie empfindsam zu sein (empathische Führung); mit Konflikten in einer Gruppe umzugehen; und eine kollaborative und partizipative Problemlösung zu ermöglichen

- Kompetenz zum kritischen Denken: die Fähigkeit, Normen, Praktiken und Meinungen zu hinterfragen; die eigenen Werte, Wahrnehmungen und Handlungen zu reflektieren; und sich im Nachhaltigkeitsdiskurs zu positionieren

Selbstkompetenz: die Fähigkeit, über die eigene Rolle in der lokalen Gemeinschaft und (globalen) Gesellschaft nachzudenken; kontinuierlich seine Handlungen zu bewerten und sich weiter zu motivieren; und sich mit den eigenen Gefühlen und Wünschen auseinanderzusetzen

Integrierte Problemlösekompetenz: die übergreifende Fähigkeit, unterschiedliche Problemlösungsrahmen für komplexe Nachhaltigkeitsprobleme anzuwenden und passfähige, inklusive und gerechte Lösungsmöglichkeiten zu entwickeln, die eine nachhaltige Entwicklung fördern und die oben genannten Kompetenzen integrieren

Quelle: UNESCO, 2017 
tenzen (Rychen, 2003; Weinert, 2001), die es ihnen ermöglichen, konstruktiv und verantwortungsvoll mit der heutigen Welt umzugehen. Einige Schlüsselkompetenzen werden im internationalen BNE-Diskurs als besonders wichtig für eine nachhaltige Entwicklung angesehen (Abb. 3) (de Haan, 2010; de Haan et al., 2008; Rieckmann, 2012, 2013, 2018; Wiek et al., 2011, 2016).

Die Nachhaltigkeitskompetenzen machen deutlich, was Nachhaltigkeitsbürger/-innen insbesondere benötigen, um sich mit den heutigen komplexen Herausforderungen auseinanderzusetzen. Sie sind für alle SDGs relevant und ermöglichen es den Individuen, die verschiedenen SDGs in Beziehung zueinander zu setzen, um das große Ganze der Agenda 2030 für Nachhaltige Entwicklung zu erkennen (UNESCO, 2017).

\section{Spezifische Lernziele für die SDGs}

Während die Nachhaltigkeitskompetenzen dazu befähigen, insgesamt mit den SDGs umzugehen, lässt sich für jedes einzelne SDG die Frage stellen, wie damit verbundene, spezifische Lernprozesse gestaltet werden können.

Dazu bietet der von der UNESCO (2017) veröffentliche Leitfaden „Education for Sustainable Development Goals. Learning Objectives“ vielfältige Anregungen. Dieses Dokument zielt darauf ab, politische Entscheidungsträger/innen, Curriculum-Entwickler/innen und Lehrende bei der Gestaltung von Strategien, Lehrplänen und Bildungsveranstaltungen zu unterstützen, um Lernen für die SDGs zu fördern. Im Sinne einer allgemeinen Orientierung sind die Lernziele, Themen und Aktivitäten für jedes SDG, nicht für spezifische Altersgruppen, Lernumgebungen oder bestimmte soziokulturelle Kontexte, zugeschnitten. Sie sind so konzipiert, dass sie für alle Lernenden aller Altersgruppen weltweit relevant sind und ihre Anwendung in allen möglichen Lernumgebungen finden können. Es handelt sich also um Vorschläge für Lernziele und -inhalte, die Pädagoginnen und Pädagogen auswählen und an ihre konkreten Lehr-Lern-Kontexte (soziokulturelle Anforderungen, Zielgruppen, etc.) anpassen können. Es werden für jedes SDG Lernziele in einer kognitiven, sozioemotionalen und verhaltensbezogenen Domäne beschrieben (UNESCO, 2017):

- Die kognitive Domäne umfasst Wissen und kognitive Fähigkeiten, die notwendig sind, um das SDG und die mit seinem Erreichen verbundenen Herausforderungen besser zu verstehen.

- Die sozioemotionale Domäne umfasst soziale Kompetenzen, die es Lernenden ermöglichen, zusammenzuarbeiten, zu verhandeln und zu kommunizieren, um das SDG voranzubringen, sowie Selbstreflexionsfähigkeiten, Werte, Einstellungen und Motivationen, die es den Lernenden ermöglichen, sich selbst weiterzuentwickeln.

- Die verhaltensbezogene Domäne beschreibt Handlungskompetenzen.

- Darüber hinaus werden für jedes SDG beispielhafte Themen und pädagogische Ansätze skizziert.

Zur Verdeutlichung dieses Ansatzes sollen hier exemplarisch die Lernziele zum SDG 1 „Keine Armut - Armut in jeder Form und überall beenden" dargestellt werden (Abb. 4).

Abb. 4: Lernziele für das SDG 1 „Keine Armut“

\begin{tabular}{|c|c|}
\hline $\begin{array}{l}\text { Cognitive learning } \\
\text { objectives }\end{array}$ & $\begin{array}{l}\text { 1. The learner understands the concepts of extreme and relative poverty and is able to critically reflect on their underlying cultural and } \\
\text { normative assumptions and practices. } \\
\text { 2. The learner knows about the local, national and global distribution of extreme poverty and extreme wealth. } \\
\text { 3. The learner knows about causes and impacts of poverty such as unequal distribution of resources and power, colonization, conflicts, } \\
\text { disasters caused by natural hazards and other climate change-induced impacts, environmental degradation and technological disa- } \\
\text { sters, and the lack of social protection systems and measures. } \\
\text { 4. The learner understands how extremes of poverty and extremes of wealth affect basic human rights and needs. } \\
\text { 5. The learner knows about poverty reduction strategies and measures and is able to distinguish between deficit-based and strength-ba- } \\
\text { sed approaches to addressing poverty. }\end{array}$ \\
\hline $\begin{array}{l}\text { Socio-emotional } \\
\text { learning objectives }\end{array}$ & $\begin{array}{l}\text { 1. The learner is able to collaborate with others to empower individuals and communities to affect change in the distribution of power } \\
\text { and resources in the community and beyond. } \\
\text { 2. The learner is able to raise awareness about extremes of poverty and wealth and encourage dialogue about solutions. } \\
\text { 3. The learner is able to show sensitivity to the issues of poverty as well as empathy and solidarity with poor people and those in vulne- } \\
\text { rable situations. } \\
\text { 4. The learner is able to identify their personal experiences and biases with respect to poverty. } \\
\text { 5. The learner is able to critically reflect on their own role in maintaining global structures of inequality. }\end{array}$ \\
\hline $\begin{array}{l}\text { Behavioural learning } \\
\text { objectives }\end{array}$ & $\begin{array}{l}\text { 1. The learner is able to plan, implement, evaluate and replicate activities that contribute to poverty reduction. } \\
\text { 2. The learner is able to publicly demand and support the development and integration of policies that promote social and economic } \\
\text { justice, risk reduction strategies as well as poverty eradication actions. } \\
\text { 3. The learner is able to evaluate, participate in and influence decision-making related to management strategies of local, national and } \\
\text { international enterprises concerning poverty generation and eradication. } \\
\text { 4. The learner is able to include poverty reduction, social justice and anti-corruption considerations in their consumption activities. } \\
\text { 5. The learner is able to propose solutions to address systemic problems related to poverty. }\end{array}$ \\
\hline
\end{tabular}


Um dieses Wissen und die genannten Fähigkeiten bei den Lernenden zu entwickeln, bieten sich einige Inhalte besonders an (Abb. 5). Dabei handelt es sich um keine erschöpfende oder endgültige Auflistung von Themen, sondern um Vorschläge von weltweit relevanten Aspekten des jeweiligen SDG. Diese sollten durch jeweils lokal relevante Themen ergänzt und mit neuen Themen aktualisiert werden, die sich ständig in unserer sich schnell verändernden Welt ergeben.

Abb. 5: Vorschläge für Themen zur Bearbeitung des SDG 1 „Keine Armut“

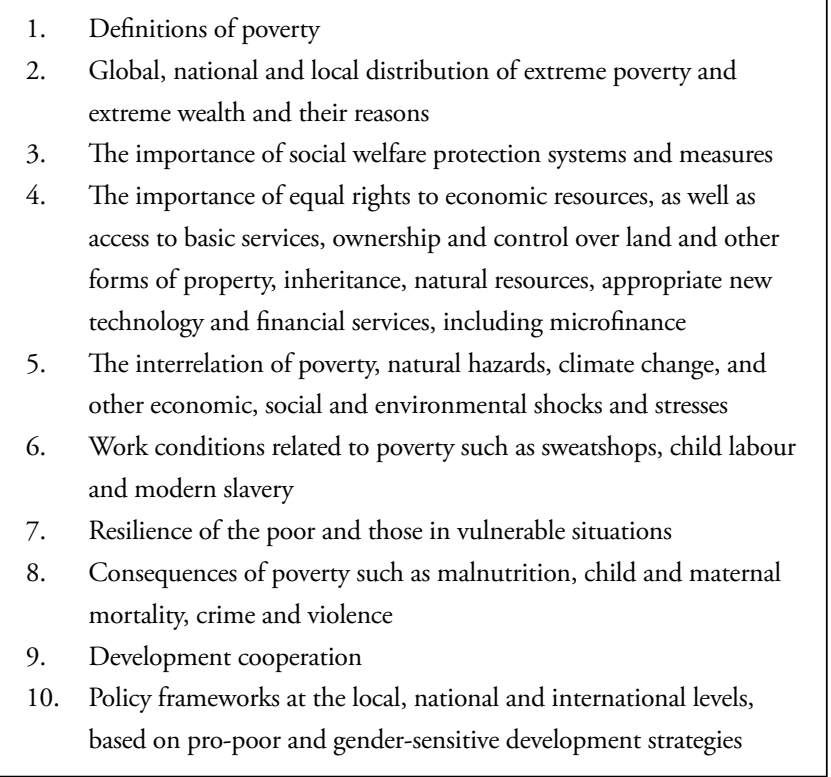

Quelle: UNESCO, 2017, S. 13

Ebenso werden einige Methoden vorgestellt, die - neben vielen anderen möglichen Methoden - zur Bearbeitung der Themen und der Entwicklung der jeweiligen Lernziele geeignet sind (Abb. 6).

Die für jedes SDG beschriebenen, spezifischen Lernziele sind immer in Verbindung mit den übergreifenden Nachhaltigkeitskompetenzen zu sehen. Denn erst die Schlüsselkompetenzen ermöglichen es den Lernenden, die komplexen Zusammenhänge und auch Widersprüche zwischen den 17 SDGs zu erkennen und zu verstehen.

\section{Handlungsorientierte transformative Pädagogik}

Für die Entwicklung der Nachhaltigkeitskompetenzen und das Erreichen der Lernziele für die einzelnen SDGs bedarf es einer handlungsorientierten, transformativen Pädagogik, die sich durch Aspekte wie selbstgesteuertes Lernen, Partizipation und Kooperation, Problemorientierung, Inter- und Transdisziplinarität und die Verknüpfung von formalem und informellem Lernen auszeichnet (UNESCO, 2017). Nur solche pädagogischen Ansätze ermöglichen den Erwerb derjenigen Schlüsselkompetenzen, die für die Förderung einer nachhaltigen Entwicklung erforderlich sind (Rieckmann, 2016, 2018). Einer Bildung für nachhaltige Entwicklung geht es darum, die Lernenden zu befähigen und zu motivieren, aktive Nachhaltigkeitsbürger/innen zu werden, die in der Lage sind, kritisch zu denken und sich an der Gestaltung einer nachhaltigen Zukunft zu beteiligen. Pädagogische
Abb. 6: Beispielhafte Lernansätze und-methoden für das SDG 1 „Keine Armut"

1. Develop partnerships between schools and universities in different regions of the world (South and North; South and South).

2. Plan and run an awareness campaign about poverty locally and globally.

3. Plan and run a student company selling fair trade products.

4. Plan and implement local service-learning and/or engagement opportunities for empowering poor people, reducing their vulnerability to different hazards and increasing their resilience - in collaboration with NGOs, the private sector and/or community groups, etc.

5. Conduct a case study on poverty and wealth in selected countries (through desktop research) or at the local level (through excursions, doing interviews, etc.).

6. Provide internships within organisations addressing poverty.

7. Develop an enquiry-based project around: "Is poverty increasing or decreasing?"

Quelle: UNESCO, 2017, S. 13

Ansätze, die diesem Ziel entsprechen, sind lernerzentriert, handlungsorientiert und transformativ (Abb. 7). Diese pädagogischen Ansätze stellen allgemeine Prinzipien für die Gestaltung von Lernprozessen in der BNE dar. Spezifische Methoden, die diesen Prinzipien entsprechen, sind erforderlich, um den Lernprozess zu ermöglichen.

In der BNE werden Methoden bevorzugt, die die Entwicklung von Kompetenzen durch aktives Lernen fördern. Bestimmte Methoden können für BNE besonders empfohlen werden (Abb. 8). Diese partizipativen Lehr- und Lernmethoden befähigen die Lernenden, sich an der Gestaltung einer nachhaltigen Entwicklung zu beteiligen. Um vielfältige und grenzüberschreitende Lernumgebungen zu schaffen und umfassende Bilder der SDGs zu entwickeln, sollten Bildungseinrichtungen und Lehrende Partnerschaften auf lokaler, nationaler und internationaler Ebene fördern (UNESCO, 2017). Es ist wichtig zu erkennen, dass angemessene Reaktionen auf Nachhaltigkeitsprobleme nicht auf einzelne Perspektiven, Disziplinen oder Arten des Wissens beschränkt werden können. Partnerschaften, die eine Reihe von gesellschaftlichen Akteuren wie Unternehmen, NGOs, öffentliche Institutionen, politische Entscheidungsträger und/oder Einzelpersonen einbeziehen, eröffnen neue Lernmöglichkeiten. In einem Dialog oder einem Projekt, das die Zusammenarbeit mit einem Praxispartner beinhaltet, können die Lernenden etwas über realweltliche Herausforderungen erfahren und von den Kompetenzen und Erfahrungen der Partner profitieren (Barth, Adomßent, Fischer, Richter \& Rieckmann, 2014). Partnerschaften zwischen Lernenden aus der ganzen Welt fördern den Austausch unterschiedlicher Perspektiven und Kenntnisse zu demselben Thema. Zum Beispiel können virtuelle Lehrveranstaltungen eine Umgebung schaffen, um einen globalen Dialog zu üben und gegenseitigen Respekt und Verständnis zu fördern (Barth \& Rieckmann, 2009).

\section{Fazit}

Für das Erreichen der SDGs kommt BNE eine wichtige Bedeutung zu. Sie kann die Entwicklung von Nachhaltigkeitskompe- 
Abb. 7: Zentrale pädagogische Ansätze einer BNE

\begin{abstract}
Lernerzentrierte Ansätze
Die lernerzentrierte Pädagogik geht von selbstständigen Lernenden aus und betont die aktive Entwicklung des Wissens anstelle eines bloßen Wissenstransfers und/oder passiven Lernerfahrungen. Das Vorwissen der Lernenden sowie ihre Erfahrungen im sozialen Kontext sind Ausgangspunkte für die Förderung von Lernprozessen, in denen die Lernenden ihre eigene Wissensbasis konstruieren. Lernerzentrierte Ansätze erfordern von den Lernenden, über ihre eigenen Kenntnisse und Lernprozesse nachzudenken, um sie zu steuern und zu verfolgen. Lehrende sollten diese Reflexionen anregen und unterstützen. Lernerzentrierte Ansätze verändern die Rolle der/des Lehrenden hin zu Moderator/inn/en von Lernprozessen (Barth, 2015).

Handlungsorientiertes Lernen

Im handlungsorientierten Lernen werden die Lernenden selbst tätig und reflektieren ihre Erfahrungen in Bezug auf den beabsichtigten Lernprozess und die angestrebte persönliche Entwicklung. Diese Erfahrungen könnten sich aus einem Projekt, einem Praktikum, der Durchführung eines Workshops, einer Kampagne etc. ergeben. Handlungsorientiertes Lernen bezieht sich auf Kolbs Theorie des erfahrungsbasierten Lernzyklus (vgl. weiterführend Kolb, 1984) und steigert den Wissenserwerb, die Kompetenzentwicklung und die Klärung von Werten durch die Verknüpfung von abstrakten Konzepten mit persönlicher Erfahrung und dem Leben des Lernenden. Die Rolle des/der Lehrenden besteht darin, eine Lernumgebung zu schaffen, die die Erfahrungen der Lernenden und reflexive Denkprozesse anregt.

Transformatives Lernen

Das transformative Lernen zielt darauf $a b$, die Lernenden dazu zu befähigen, die Art und Weise zu hinterfragen und zu verändern, wie sie die Welt sehen und über sie denken, um ihr Verständnis von ihr zu erweitern (Slavich \& Zimbardo, 2012; Mezirow, 2000). Der/die Lehrende ist ein Facilitator, der die Lernenden befähigen soll, ihre Weltanschauungen zu verändern. Das verwandte Konzept des transgressiven Lernens (Lotz-Sisitka, Wals, Kronlid \& McGarry, 2015) geht noch einen Schritt weiter: Es betont, dass das Lernen in der BNE den Status quo überwinden und die Lernenden für disruptives (störendes) Denken und die Co-Kreation neuen Wissens vorbereiten sollte.
\end{abstract}

Quelle: UNESCO, 2017, S. 12

tenzen fördern, die Individuen nicht nur dazu befähigen, die SDGs in ihrer ganzen Komplexität zu verstehen, sondern auch eigene Beiträge zum Erreichen der Ziele zu leisten. Gleichzeitig kann BNE auch spezifische Lernprozesse ermöglichen, die dazu benötigt werden, sich ein bestimmtes SDG zu erschließen und eigene Beiträge zu diesem Ziel zu leisten. Zudem bieten die SDGs eine inhaltliche Orientierung für BNE. Sie zeigen wesentliche Themenstellungen auf, die es im Rahmen einer BNE zu bearbeiten gilt. Für die Entwicklung der Nachhaltigkeitskompetenzen und eine Auseinandersetzung mit den einzelnen SDGs bedarf es einer handlungsorientierten, transformativen Pädagogik. Jedoch sollte BNE auch Räume für eine kritische Reflexion über die SDGs eröffnen. So kann es z.B. darum gehen, mit Lernenden die den SDGs inhärenten Widersprüche (u.a. in Bezug auf Wirtschaftswachstum, Messner \& Scholz, 2015) bzw. Leerstellen (u.a. „Dekarbonisierung“, „planetare Grenzen“, „Benennung und Bearbeitung von Ursachen hinter den zu lösenden
Problemlagen“, Messner \& Scholz, 2015, S. 23; Scholz, 2017) zu analysieren und zu diskutieren. Eine nachhaltige Entwicklung selbst ist ein Lernprozess, und Bildung für nachhaltige Entwicklung - verstanden als emanzipatorisches Bildungskonzept sollte dazu beitragen, die Ziele und Grundlagen einer nachhaltigen Entwicklung immer wieder zu hinterfragen und weiterzuentwickeln.

Abb. 8: Schlüsselmethoden für das Lernen der SDGs

- Kollaborative Projekte in Ernstsituationen wie Service-LearningProjekte und Kampagnen für verschiedene SDGs

- Methoden zur Entwicklung von Zukunftsentwürfen wie Zukunftswerkstätten, Szenarioanalysen, utopische/dystopische Erzählungen, Science-Fiction-Denken sowie Prognosen und Backcasting

- Analysen komplexer Systeme durch partizipative Forschungsprojekte, Fallstudien, Stakeholder-Analysen, Akteursanalysen, Modellierung, Systemspiele, etc.

- Kritisches und reflektierendes Denken durch Fisch-Bowl-Diskussionen, Lerntagebücher, etc.

Quelle: UNESCO, 2017

Um mit BNE zur Förderung der SDGs beizutragen und sie aber auch kritisch zu reflektieren, ist es allerdings notwendig, dass BNE als Querschnittsanliegen in alle Bildungsbereiche integriert wird. Dieser Anspruch wird im Nationalen Aktionsplan Bildung für nachhaltige Entwicklung (Nationale Plattform Bildung für nachhaltige Entwicklung, 2017) formuliert und es sind in den letzten Jahren Entwicklungen in diese Richtung zu verzeichnen gewesen.

Weiterhin stellt es aber eine große Herausforderung dar, BNE als orientierendes Bildungskonzept in den Strukturen und der Praxis des Bildungssystems zu verankern.

\section{Literatur}

Barth, M. (2015). Implementing sustainability in higher education: learning in an age of transformation. London: Routledge.

Barth, M., Adomßent, M., Fischer, D., Richter, S. \& Rieckmann, M. (2014) Learning to change universities from within: a service-learning perspective on promoting sustainable consumption in higher education. Journal of Cleaner Production, 62, 72-81.

Barth, M. \& Rieckmann, M. (2009). Experiencing the Global Dimension of Sustainability: Student Dialogue in a European-Latin American Virtual Seminar. International Journal of Development Education and Global Learning, 1(3), 23-38.

de Haan, G. (2010). The development of ESD-related competencies in supportive institutional frameworks. International Review of Education, 56(2), 315-328.

de Haan, G., Kamp, G., Lerch, A., Martignon, L., Müller-Christ, G. \& Nutzinger, H.-G. (Hrsg.) (2008). Nachhaltigkeit und Gerechtigkeit. Grundlagen und schulpraktische Konsequenzen. Berlin \& Heidelberg: Springer.

Kercher, J. (2015). Wie die Nachhaltigkeitsziele in die Welt kamen. Von den MDGs zu den SDGs. In oekom e.V. - Verein für ökologische Kommunikation (Hrsg.), Nachhaltige Entwicklungsziele. Agenda für eine bessere Welt? (S. 27-33). München: Oekom.

Kolb, D. A. (1984). Experiential Learning: Experience as the Source of Learning and Development. Englewood Cliffs, N.J.: Prentice-Hall.

Lotz-Sisitka, H., Wals, A. E., Kronlid, D. \& McGarry, D. (2015). Transformative, transgressive social learning: rethinking higher education pedagogy in times of systemic global dysfunction. Current Opinion in Environmental Sustainability, 16, 73-80.

Messner, D. \& Scholz, I. (2015). Gemeinsam für das Wohlergehen aller. Agenda 2030 und die Sustainable Development Goals. In oekom e.V. - Verein für ökologische Kommunikation (Hrsg.), Nachhaltige Entwicklungsziele. Agenda für eine bessere Welt? (S. 18-26)München: Oekom. 
Mezirow, J. (2000). Learning as transformation: critical perspectives on a theory in progress. San Francisco: Jossey-Bass.

Nationale Plattform Bildung für nachhaltige Entwicklung (2017). Nationaler Aktionsplan Bildung für nachhaltige Entwicklung. Der deutsche Beitrag zum UNESCOWeltaktionsprogramm. Zugriff am 18.05.2018 http://www.bne-portal.de/sites/ default/files/Nationaler_Aktionsplan_BNE_2017.pdf

Overwien, B. (2013). Kompetenzmodelle im Lernbereich „Globale Entwicklung“ - Bildung für nachhaltige Entwicklung. In B. Overwien \& H. Rode (Hrsg.), Bildung für nachhaltige Entwicklung. Lebenslanges Lernen, Kompetenz und gesellschaftliche Teilhabe (S. 13-34). Leverkusen: Budrich.

Rieckmann, M. (2012). Future-oriented higher education: Which key competencies should be fostered through university teaching and learning? Futures, 44(2), $127-135$.

Rieckmann, M. (2013). Schlüsselkompetenzen für eine nachhaltige Entwicklung. POLIS, 2013(4), 11-14.

Rieckmann, M. (2018). Chapter 2 - Learning to transform the world: key competencies in ESD. In A. Leicht, J. Heiss \& W. J. Byun (Hrsg.), Issues and trends in Education for Sustainable Development (S. 39-59). Paris: UNESCO.

Rieckmann, M. \& Schank, C. (2016). Sozioökonomisch fundierte Bildung für nachhaltige Entwicklung - Kompetenzentwicklung und Werteorientierungen zwischen individueller Verantwortung und struktureller Transformation. SOCIENCE, 1(1), 65-79.

Rychen, D. S. (2003). Key competencies: Meeting important challenges in life. In D. S. Rychen \& L. H. Salganik (Hrsg.), Key competencies for a successful life and well-functioning society (S. 63-107). Cambridge/ MA: Hogrefe and Huber.

Scholz, I. (2015). Universelle Ziele für eine nachhaltige Entwicklung. In: H. Leitschuh, G. Michelsen, U. E. Simonis, J. Sommer \& E. U. von Weizsäcker (Hrsg.), Gesucht: Weltumweltpolitik. Herausforderungen im Anthropozän (S. 116-125). Stuttgart: Hirzel.

Scholz, I. (2017). Herausforderung Sustainable Development Goals. In G. Michelsen (Hrsg.), Die deutsche Nachhaltigkeitsstrategie. Wegweiser für eine Politik der Nachhaltigkeit (S. 23-39). Wiesbaden: Hessische Landeszentrale für politische Bildung Slavich, G. M. \& Zimbardo, P. G. (2012). Transformational Teaching: Theoretical Underpinnings. Basic Principles, and Core Methods. Educational Psychology Review, 24(4), 569-608.

UNESCO (2014): UNESCO Roadmap zur Umsetzung des Weltaktionsprogramms „Bildung für nachhaltige Entwicklung". Zugriff am 18.05.2018 https://www.bmbf. de/files/2015_Roadmap_deutsch.pdf

UNESCO (2015a): Global Citizenship Education: Topics and learning objectives. Zugriff am 18.05.2018 http://unesdoc.unesco.org/images/0023/002329/232993e.pdf
UNESCO (2015b): Rethinking Education. Towards a global common good? Zugriff am 18.05.2018 http://unesdoc.unesco.org/images/0023/002325/232555e.pdf

UNESCO (2017). Education for Sustainable Development Goals. Learning Objectives. Zugriff am 18.05.2018 http://unesdoc.unesco.org/images/0024/002474/ $247444 \mathrm{e}$ pdf

Vereinte Nationen (2015). Transformation unserer Welt: die Agenda 2030 für nachhaltige Entwicklung. Zugriff am 18.05.2018 http://www.un.org/depts/german/gv70/band1/ar70001.pdf

Wals, A. E. J. (2015). Beyond unreasonable doubt. Education and learning for socio-ecological sustainability in the Anthropocene. Zugriff am 18.05.2018https://arjenwals. files.wordpress.com/2016/02/8412100972_rvb_inauguratie-wals_oratieboekje_ v02.pdf, Abruf: 18.05.2018

Wals, A. E. J. \& Lenglet, F. (2016). Sustainability citizens: Collaborative and disruptive social learning. In R. Horne, J. Fien, B. B. Beza \& A. Nelson (Hrsg.), Sustainability Citizenship in Cities: Theory and Practice (S. 52-67). London: Routledge.

Weinert, F. E. (2001). Concept of Competence: A Conceptual Clarification. In D. S. Rychen \& L. H. Salganik (Hrsg.), Defining and Selecting Key Competencies (S. 45-65). Seattle, Toronto, Bern \& Göttingen: Hogrefe und Huber.

Wiek, A., Withycombe, L. \& Redman, C. L. (2011): Key competencies in sustainability: a reference framework for academic program development. Sustainability Science, 6(2), 203-218.

Wiek, A., Bernstein, M. J., Foley, R. W., Cohen, M., Forrest, N., Kuzdas, C., Kay, B. \& Withycombe Keeler, L. (2016): Operationalising competencies in higher education for sustainable development. In M. Barth, G. Michelsen, I. Thomas \& M. Rieckmann (Hrsg.), Routledge Handbook of Higher Education for Sustainable Development (S. 241-260). London: Routledge.

\section{Prof. Dr. Marco Rieckmann}

ist Professor für Hochschuldidaktik, Schwerpunkt Schlüsselkompetenzen, im Fach Erziehungswissenschaften der Fakultät I - Bildungs- und Gesellschaftswissenschaften an der Universität Vechta. Zuvor war er wissenschaftlicher Mitarbeiter an der Leuphana Universität Lüneburg (2004-2013). Er ist Vertreter der Deutschen Gesellschaft für Erziehungswissenschaft (DGfE) im Council der European Educational Research Association (EERA) sowie Sprecher des Deutschsprachigen Netzwerks „LehrerInnenbildung für nachhaltige Entwicklung" (LeNa). Seine Arbeitsschwerpunkte sind: Hochschuldidaktik, (Hochschul-)Bildung für nachhaltige Entwicklung, Nachhaltige Hochschulentwicklung. 